Original article

\title{
Evaluation of adherence to oxygen therapy standards before, during and after oxygen hood administration
}

\author{
Zeinab Kord $^{1 *}$, Shahriar Salehi Tali², Fatemeh Alaee Karharoudy ${ }^{3}$
}

(Received: 7 Jul 2015; Accept: 5 Oct 2015)

\begin{abstract}
Background and Purpose: Oxygen therapy is one of the common treatments for neonates with cardiopulmonary disease. The aim of this study was to evaluate adherence to oxygen therapy standards by nurses before, during and after using oxygen hood.

Methods: In this cross-sectional study, 250 cases of nursing care were evaluated before, during and after administration of oxygen hood therapy in preterm infants. The observations were conducted according to the standards of the neonatal intensive care units (NICU) of hospitals affiliated to the Ahvaz University of Medical Sciences. The data collection tools included a self-designed checklist for nursing audit evaluating the performance of NICU nurses working by neonatal oxygen hood, and a demographic profile checklist. The data were analyzed by Chi-square and Spearman tests, using SPSS version 18.

Results: The results demonstrated insufficient adherence to the standards of oxygen therapy by $90 \%$ of the nurses before neonatal oxygen hood. Moreover, regarding the mentioned standards, $84 \%$ and $99.2 \%$ of the nurses had moderate and poor performance during and after oxygen therapy, respectively.
\end{abstract}

Conclusion: Considering the poor performance of the NICU nurses before, during and after oxygen therapy, appropriate supervision and training programs for nurses is recommended to promote their capabilities in this regard.

Keywords: Neonatal intensive care unit, Neonatal oxygen hood, Nursing care, Preterm neonates

\section{Introduction}

Neonatal period is of great importance due to the physiological adaptations to the extrauterine environment and the high mortality of neonates indicates its sensitivity. Since infancy is one of the most critical periods of growth and development, the need for support and care in this period is more compelling than the other periods of life (1). Each year, 12.9 million preemies are born worldwide, 11 million (85\%) of whom are in Asia and Africa. Intensive care is needed to keep preterm neonates alive until organ maturation (2). Most very-low-birth weight (less than 1500 grams) premature infants with gestational age less than 28 weeks are admitted to neonatal intensive care units (NICU) (3).

Oxygen therapy is a method that provides oxygen gas at concentrations higher than environmental air. Oxygen improves cellular metabolism and improves the cardiopulmonary function. In clinical settings, oxygen should be considered as a medication; thus,

\footnotetext{
1,* Corresponding author: Faculty Member, Jundishapur University of Medical Sciences, Ahvaz, Iran. Email: Bahare.kord132@gmail.com

${ }^{2}$ School of Nursing and Midwifery, Jundishapur University of Medical Sciences, Ahvaz, Iran

${ }^{3}$ School of Nursing and Midwifery, Shahid Beheshti University of Medical Sciences, Tehran, Iran
} 
precautionary measures must be monitored during oxygen therapy $(4,5)$.

Oxygen is essential to man life and necessary to detoxify poisonings interfering with the blood or tissue oxygenation (6). Low hemoglobin oxygen saturation in premature newborns causes chronic hypoxia, which leads to several complications including growth failure, central nervous system (CNS) damages, cardiovascular and chronic pulmonary diseases and finally impaired development of nervous system and mortality in the first weeks of life (7).

Oxygen toxicity is a condition resulting from long-term (over 48 hours) exposure to oxygen with high concentration (over 50\%), which accelerates occurrence of convulsion, pulmonary damage, retrolental fibroplasia and CNS injuries $(8,9)$.

Oxygen therapy is a common practice in premature or hospitalized neonates' care. Oxygen is a therapeutic agent mostly used in NICUs, and similar to any other medical intervention its complications, administration and home monitoring after discharge must be taken into account. In order to manage oxygen therapy complications and proper administration, the standards of care must be applied $(10,11)$. Oxygen therapy elevates blood oxygen and improves the cardiopulmonary function by supplying enough oxygen to the tissues and inhibiting the accumulation of lactic acid (9). Oxygen therapy is administered through various modalities, one of which is neonatal oxygen hood, which is a plastic dome fitting over an infant's head and neck, and supplying warm and moist oxygen (12).

Rood-dehghan investigated oxygen therapy performance of 36 nurses in the respiratory disease wards of hospitals affiliated to Tehran University of Medical Sciences using observation and interviews. The results of this study demonstrated that the performance of all the nurses before oxygen therapy at both times of observation (100\%) and during oxygen therapy at the first time of observation $(100 \%)$ as well as the majority of the nurses during oxygen therapy at the second time of observation (96.3\%) was inappropriate (13).

A study in Greece showed that $41 \%$ of head nurses did not consider oxygen as a therapeutic but as a supplemental action for better outcomes. Moreover, most of the head nurses believed that oxygen could improve dyspnea in patients. Approximately $88 \%$ of the head nurses had not established any oxygen therapy protocols in their wards. In $57 \%$ of the cases, adherence to the medical guidelines of oxygen therapy was suggested. In the absence of such guidelines, $6.96 \%$ of the nurses decided to administer oxygen therapy and in other cases, physicians were consulted about oxygen therapy (14).

Intensive care units (ICU) share the most important problems of health care systems. These problems include inappropriate patient care, ineffective communication between medical staff and patients, lack of expression of differences between healthcare workers' performances by patients and numerous other deficiencies, which might result in increased mortality rate (15). Management and monitoring the oxygen flow provides detailed information for the nurses and helps with decision-making. It also establishes a criterion for assessing and monitoring the quality of nursing care in hospitals and other health centers (16).

Oxygen therapy is an important responsibility of the nurses for maintenance of the patients' safety. Since numerous preterm neonates in NICUs undergo oxygen therapy, and given the importance of oxygen therapy care, this study was performed to audit the quality of oxygen therapy care. In so doing, we evaluated the adherence to the standards of oxygen therapy by nurses before, during and after neonatal oxygen hood administration at our hospitals.

\section{Materials and methods}

In this cross-sectional study, we evaluated 250 cases of nursing care before, during and after neonatal oxygen hood administration in preterm neonates. The evaluations were conducted according to the standards of the NICUs of Abuzar and Imam Khomeini Hospitals affiliated to Ahvaz University of Medical Sciences from February 2012 to August 2013. The event sampling method (ESM) was used for sample collection.. Therefore, 
the researcher should be present in that situation to record observations (17). To the authors' best knowledge, no study has been conducted on this issue. The sample size, at $95 \%$ confidence level and maximum error of 0.05 , was calculated to be 250 .

Nurses' demographics such as age, occupational experience, level of education, marital status, working shift and family size, as well as information on attending subspecialty and newborn care training courses were collected using a questionnaire.

The data on the nursing activities associated with neonatal oxygen hood administration in preemies were collected using a checklist of nursing care audit pertinent to oxygen therapy in premature infants hospitalized in NICUs. The checklist was developed by the researchers based on the standards of oxygen therapy in premature infants. It contains 17, 15 and 7 statements related to before, during and after oxygen therapy, respectively (Appendix 1).

The abovementioned checklist is comprised of information on standards of neonatal oxygen therapy. It contains information on the standards of oxygen therapy care before, during and after administration of oxygen hood.

The researcher completed the oxygen therapy checklist using 'yes' (complete or incomplete) or 'no'. For 'no', 'yes, incomplete' and 'yes, complete' replies 0,1 and 2 scores were given, respectively. The checklist was completed during the observations by one of the researchers. Since attendance of the observer could affect the nurses' caring performance, it was explained to the nurses that their caring performance is observed not themselves. In addition, the checklist was not completed during the first observation and the constant presence of the observer made the practitioners feel calm and relaxed as usual.

To establish content validity of the data collection tools, the available protocols and standards as well as views of 15 nursing faculty members, neonatologists and head nurses were collected. Agreement between observations was used for determining the reliability of the checklist. For this purpose, the same checklist was given to a second observer whose skill and knowledge were quite the same as the other one. The two observers completed the checklist for ten newborns at the same time, and the correlation coefficient between the observations was calculated to be 0.96 .

At the beginning of each shift, the researcher observed nurses' performance before, during and after oxygen therapy based on the checklist. In order to complete the checklist, the nurses' care performance in oxygen therapy was recorded at the end of each procedure. The researcher attended the NICUs on a daily basis in three shifts of morning, afternoon and night, and did the structured observations in relation to oxygen therapy for preterm infants and completed the respective statement of each observation on the checklist. For instance, statements on the checklist related to before, during and after the oxygen therapy were "controls pulse oximetry", "washes his/her hands before oxygen therapy" and "reviews the lungs' sounds", respectively.

Prior to the study, we obtained the approval of the ethics committee of Shahid Beheshti University of Medical Sciences and permission of the hospital authorities by explaining the objectives and methods of the study. In addition, the researcher was introduced to the head nurses and informed consent was obtained from the participants with ensuring them of confidentiality of the data.

The data from 250 cases of oxygen therapy care for preterm infants were collected and classified as poor, moderate and good. The data were analyzed by SPSS version 18, using statistical methods to describe and analyze the data, and performing Pearson and Chi-square tests for evaluating the correlation between factors associated with neonatal nursing care.

\section{Results}

Our results demonstrated that the mean age of the nurses was $32.7 \pm 0.24$ years (age range: $23-45$ years). The means of occupational experience of the nurses in NICU and other wards were $9 \pm 8.8$ and 1 year, respectively. Additionally, $88.8 \%$ of the nurses were married and the majority of them $(50.8 \%)$ were working in the morning shift. Moreover, $80 \%$ of the NICU nurses were not trained on this issue. 
Table 1. Observance of the standards of oxygen hood administration by nurses in preterm infants in neonatal intensive care units

\begin{tabular}{|c|c|c|c|c|c|c|c|c|c|}
\hline $\begin{array}{l}\text { Oxygen therapy status } \\
\text { Oxygen therapy methods }\end{array}$ & \multicolumn{3}{|c|}{ Before oxygen therapy } & \multicolumn{3}{|c|}{ During oxygen therapy } & \multicolumn{3}{|c|}{ After oxygen therapy } \\
\hline \multirow{2}{*}{ Hood } & $\begin{array}{l}\text { Poor } \\
\mathrm{n}(\%)\end{array}$ & $\begin{array}{c}\text { Moderate } \\
\mathrm{n}(\%)\end{array}$ & $\begin{array}{l}\text { Good } \\
\mathrm{n}(\%)\end{array}$ & $\begin{array}{l}\text { Poor } \\
\mathrm{n}(\%)\end{array}$ & $\begin{array}{c}\text { Moderate } \\
\mathrm{n}(\%)\end{array}$ & $\begin{array}{l}\text { Good } \\
\mathrm{n}(\%)\end{array}$ & $\begin{array}{l}\text { Poor } \\
\mathrm{n}(\%)\end{array}$ & $\begin{array}{c}\text { Moderate } \\
\mathrm{n}(\%)\end{array}$ & $\begin{array}{r}\text { Good } \\
\mathrm{n}(\%)\end{array}$ \\
\hline & $225(90 \%)$ & $24(9.6 \%)$ & $1(0.4 \%)$ & $10(4 \%)$ & $210(84 \%)$ & $30(12 \%)$ & $248(99.2 \%)$ & $2(0.8 \%)$ & 0 \\
\hline
\end{tabular}

Table 2. The Spearman test results regarding the correlation between the observance of the standards of oxygen therapy and nurses' age and occupational experience in the neonatal intensive care units

\begin{tabular}{lcccc}
\hline $\begin{array}{l}\text { Demographics } \\
\text { Time of observation of the standards of oxygen therapy }\end{array}$ & \multicolumn{2}{c}{ Nurses' age } & \multicolumn{2}{c}{ Nurses' occupational experience } \\
\cline { 2 - 5 } Before oxygen therapy & P-value & r & 0.57 & 0.001 \\
\hline During oxygen therapy & 0.001 & 0.33 & 0.5 & 0.001 \\
\hline After oxygen therapy & 0.001 & 0.33 & 0.001 \\
\hline
\end{tabular}

The results underscore poor performance of the NICU nurses in the observance of the standards of neonatal oxygen hood administration in $90 \%$ of the cases before oxygen therapy. Additionally, $84 \%$ and $99.2 \%$ of the performance of the nurses scored moderate and poor during and after oxygen therapy, respectively (Table 1).

The statistical analyses reflected no significant relationships between the oxygen therapy care performance of the nurses and their marital status $(\mathrm{P}=0.18)$, working shift $(\mathrm{P}=0.4)$, subspecialty nursing courses $(\mathrm{P}=1.07)$ and academic education $(\mathrm{P}=0.2)$. The Spearman correlation test showed no significant relationships between occupational experience in wards other than NICU $(\mathrm{P}=1.3)$ and attending neonatal care training courses $(\mathrm{P}=0.5)$. However, a significant relationship was observed between the nurses' oxygen therapy care performance and their age $(\mathrm{P}=0.001)$ and occupational experience in NICU $(\mathrm{P}=0.001)$ (Table 2).

\section{Discussion}

This study aimed to evaluate the NICU nurses' adherence to the standards of oxygen therapy before, during and after oxygen hood administration. Our findings illustrate that the observance of the standards before oxygen hood therapy in $90 \%$ of cases was poor.

This finding is consistent with a similar study in Iran, in which observance of the standards before oxygen hood therapy in all cases was poor (13). Knowledge of nurses has an important role in patient care. Performance of nurses who are certified in critical care should be assessed regularly to ensure their satisfactory performance. Providing clinical critical care for preterm infants requires a holistic view. Intensive care has sensible and observable characteristic that should be monitored in nursing activities. In other words, evaluation of the quality of nursing care is a step towards the management of nursing activities, which can promote nurses' performance in hospitals and other healthcare centers (18).

Our results showed that $80 \%$ of the nurses in this study were not trained. However, the results of a study conducted in Virginia, the USA, indicated that all NICU nurses had passed specialized training courses in neonatal intensive care (19). Whereas in a study performed in Iran $73.8 \%$ of NICU nurses had not received any specialized training (18).

Due to technological advancements of NICUs, for the nurses to reach their full potential and to promote the quality of nursing care, the scientific and practical knowledge of nurses should be improved. Thus, providing regular training programs for nurses seems to be necessary to keep them up to date on the nursing practice and new technology (20).

Training courses can promote nurses' awareness and performance, especially in NICUs, which in turn, results in provision of high quality services (21). A study carried out in Ontario, Canada, on 3408 nursing graduates working in two large 
teaching hospitals showed that over half of the nurses required further scientific and practical knowledge for proper accomplishment of their duties (22).

The possible reasons for the poor observance of the standards before oxygen therapy might be associated with the following factors: absence of proper oxygen therapy protocols, insufficient knowledge of nurses about oxygen therapy in preterm neonates' care, inadequate supervision on adherence to oxygen therapy standards, lack of continuous medical education courses for NICU nurses and absence of physician prescription of monitoring breathing sounds, chest X-ray and arterial blood gas.

Nurses' performance can be enhanced by providing training courses and oxygen therapy protocols. The nurses caring for preemies must act in accordance with the scientific methods of oxygen therapy, provide the necessary training for parents of neonates under oxygen therapy and record vital signs while using oxygen as any other therapeutic (23).

Provision of effective health care needs assessment and all types of assessments have a necessary prerequisite called standard. Lack of standards makes any assessment unreliable, and precise and well-defined standards ensure accurate assessment. Absence of comprehensive, precise and clear standards is one of the reasons for inefficiency of hospitals (24). To provide adequate assessment an appropriate and standardized checklist is required.

In Iran, Ministry of Health and Medical Education is responsible for developing standards. Consistent with the local conditions, the standards should also be developed according to the principles of the International Organization of Standards and some other standards such as the Joint Commission on Accreditation of Healthcare Organizations (25). Nowadays, establishing standards for all the nursing activities is an unresolved issue. Standardized clinical practice guidelines systematically propose nursing interventions, based on different nursing requirements so that nurses can improve the quality of care and reduce the ensuing costs (26). This study can be the basis for the standardization of nursing care in oxygen therapy.

The results of this study along with the standard guidelines on oxygen therapy nursing care can identify and minimize the weaknesses and as a result, provide the optimal level of care. In addition, insufficient knowledge on oxygen therapy should be addressed through specialized training programs and establishing standard care principles.

\section{Conclusion}

The NICU nurses' observance of the standards of oxygen therapy in preterm infants before and after oxygen therapy was poor; while, during oxygen therapy the score was moderate. With regard to the absence of a comprehensive standard checklist for oxygen therapy, the results of this study can be the basis for standardization of the oxygen therapy nursing care and provision of a checklist.

\section{Conflict of interest}

None declared.

\section{Author's contributions}

$\mathrm{Z}$ Kord and Sh Salehi contributed to the designing, writing, revising, and approving the final manuscript. L Khanali performed data collection. F Alaee was involved in the study conception, design, and material support. H Alijani reviewed the content and provided revisions.

\section{Acknowledgements}

This article is part of an MSc thesis entitled "Audit of nursing care activities associated with oxygen hood therapy method in premature infants hospitalized in the neonatal intensive care units of hospitals affiliated to Ahvaz University of Medical Sciences" approved and conducted in Shahid Beheshti University of Medical Sciences of Tehran, Iran. We would like to thank all the nurses who cooperated with this study. 


\section{Reference}

1. Clark RL, Lui K, Oei JL. Use of oxygen in the resuscitation of preterm infants: current opinion and practice in Australia and New Zealand. J Paediatr Child Health 2009; 45(1-2):31-5.

2. Jean AD, Stack DM. Full-term and very-low-birth-weight preterm infants' self-regulating behaviors during a StillFace interaction: influences of maternal touch. Infant Behav Dev 2012; 35(4):779-91.

3. Martin CR, Brown YF, Ehrenkranz RA, O'Shea TM, Allred EN, Belfort MB, et al. Nutritional practices and growth velocity in the first month of life in extremely premature infants. Pediatrics 2009; 124(2):649-57.

4. Manja V, Lakshminrusimha S, Cook DJ. Oxygen saturation target range for extremely preterm infants: a systematic review and meta-analysis. JAMA Pediatr 2015; 169(4):332-40.

5. Toriyama M, Ishiguro A, Motojima Y, Yamana K, Sobajima H, Tamura M. Oxygen requirement and surfactant therapy in preterm infants after delivery. Pediatr Int 2015; 57(1):55-9.

6. Johnson K, Scott SD, Fraser KD. Oxygen use for preterm infants: factors that may influence clinical decisions surrounding oxygen titration. Adv Neonatal Care 2011; 11(1):8-14.

7. Deuber C, Terhaar M. Hyperoxia in very preterm infants: a systematic review of the literature. J Perinat Neonatal Nurs 2011; 25(3):268-74.

8. Nishimura M. High-flow nasal cannula oxygen therapy in adults. J Intensive Care 2015; 3(1):15.

9. Chanques G, Contantin JM, Sauter M, Jung B, Sebbane M, Verzilli D, et al. Discomfort associated with underhumidified high-flow oxygen therapy in critically ill patients. Intensive Care Med 2009; 35(6):996-1003.

10. Parke RL, Eastwood GM, McGuinness SP; George Institute for Global Health; Australian and New Zealand Intensive Care Society Clinical Trials Group. Oxygen therapy in non-intubated adult intensive care patients: a point prevalence study. Crit Care Resusc 2013; 15(4):287-93.

11. Fealy N, Osborne C, Eastwood GM, Glassford N, Hart G, Bellomo R. Nasal high-flow oxygen therapy in ICU: A before-and-after study. Aust Crit Care 2015. pii: S10367314(15)00069-7.

12. Ramachandrappa A, Jain L. Elective cesarean section: its impact on neonatal respiratory outcome. Clin Perinatol 2008; 35(2):373-vii.

13. Rood-dehghan Z, Shaban M, Memari A, Mehran A. Staff nurses' adherence to oxygen therapy standards. IJN 2011; 23(68):8-18 (Persian).

14. Brokalaki H, Matziou V, Zyga S, Kapella M, Tsaras K, Brokalaki E, et al. Omissions and errors during oxygen therapy of hospitalized patients in a large city of Greece. Intensive Crit Care Nurs 2004; 20(6):352-7.

15. Terblanche M, Adhikari NKJ. The evolution of intensive care unit performance assessment. J Crit Care 2006; 21(1):19-22.

16. Justham D. Measuring performance through audit projects in orthopaedics-how do you know your performance is up to standard? J Orthop Nurs 2005; 9(1):43-7.

17. Polit DF, Beck ChT. Essential of Nursing Research: Methods, Appraisal and Utilization. 7th ed. Philadelphia: Lippincott Williams \& Wilkins; 2010.

18. Goudarzi Z, Tefagh MR, Monjamed Z, Memari AM, Kamali P. The effect of continuing education of neonatal intensive care nursing on knowledge and the practice of the nurses working in the children's hospitals. Hayat 2004; 10(1):25-31 (Persian).

19. Karlowicz MG, McMurray JL. Comparison of neonatal nurse practitioners' and pediatric residents' care of extremely low-birth-weight infants. Arch Pediatr Adolesc Med 2000; 154(11):1123-6.

20. Collins JM. Reflections on the changing learning needs of nurses: a challenge for nursing continuing educators. J Contin Educ Nurs 2002; 33(2):74-7.

21. Xiao LD. Nurse educators' perceived challenges in mandatory continuing nursing education. Int Nurs Rev 2006; 53(3):217-23.

22. Butt M, Baumann A, O'Brein-Pallas L, Deber R, Blythe J, Dicenso A. The learning needs of nurses experiencing job change. J Contin Educ Nurs 2002; 33(2):67-73.

23. Bizzarro MJ, Li FY, Katz K, Shabanova V, Ehrenkranz RA, Bhandari V. Temporal quantification of oxygen saturation ranges: an effort to reduce hyperoxia in the neonatal intensive care unit. J Perinatol 2014; 34(1):33-8.

24. Kamioka H, Kawamura Y, Tsutani K, Maeda M, Hayasaka S, Okuizum H, et al. A checklist to assess the quality of reports on spa therapy and balneotherapy trials was developed using the Delphi consensus method: the SPAC checklist. Complement Ther Med 
2013; 21(4):324-32.

25. Sahebzadeh M, Bagheban G. Comparison of emergency standards approved by the Ministry of Health and Medical Education with the principles of the ISO 9001 version
2000. Iran Health Inf Manage J 2007; 3(2):103-11.

26. Galganski CJ. Mapping the literature of nursing administration. J Med Libr Assoc 2006; 94(2 Suppl):E87-E91. 